\title{
Structural health monitoring optimization for similar structures using a hierarchical Bayesian update
}

\author{
Christelle Geara $^{1,2}$, Rafic Faddoul ${ }^{1}$, Alaa Chateauneuf ${ }^{2,3}$, Wassim Raphaël ${ }^{1}$ \\ ${ }^{1}$ ESIB, Université Saint-Joseph \\ Mar Roukos, PoB. 11-154, Riad El Solh, Beyrouth, Liban \\ Christelle.geara1@usj.edu.lb; Rafic.faddoul@usj.edu.lb; Wassim.raphael@usj.edu.lb \\ ${ }^{2}$ Université Clermont Auvergne, CNRS, Institut Pascal \\ 63000 Clermont-Ferrand, France \\ ${ }^{3} \mathrm{CIDECO}$ \\ 63178 Aubière, France \\ Alaa.chateauneuf@uca.fr
}

\begin{abstract}
One of the most important issues in civil engineering is the detection of structural damages affecting the system performance at an early stage in order to prevent any catastrophic results. Two main approaches are adopted for structure monitoring: (i) periodical inspections and (ii) permanent monitoring. However, when relying on inspection results only, damages occurring between two consecutive inspections can not be detected which can be dangerous depending on the severity of the damage. Hence, for cost effective results, it is often recommended to permanently monitor structures using sensors in order to have continuous information about every single element of the structure. Since the implementation of such instruments is costly, one must optimize their configuration, number and location wise, in order to maximize the probability of detecting damages with a limited number of sensors. This configuration can be optimized, furthermore, when monitoring similar structures having at least one property in common. Thus, in this paper, we propose a methodology that optimizes the configuration of sensors for several similar structures by a genetic algorithm, and uses the obtained data in order to update, accordingly, the elements' properties (i.e. The rigidity, the Young modulus) for each structure through a hierarchical Approximate Bayesian Computation (ABC). This methodology considers all uncertainties associated with the precision of the sensors results, the mechanical model and the degradation of the elements and takes advantage of the results obtained for any structure in order to update the information of all other similar structures which could save time, costs, and give more accurate results. A numerical application on two concrete frame structures is presented to illustrate the proposed methodology.
\end{abstract}

Keywords: Structural health monitoring, Bayesian update, Genetic algorithm, Similar structures, Data fusion.

\section{Introduction}

In recent years, the structural health monitoring has been one of the most trending topics in the engineering field since certain signs can give indications that a failure is going to occur [1]. Two main approaches are adopted for structural monitoring: (i) periodical inspections and (ii) permanent continuous monitoring. While the former seems to be less costly, an obvious drawback is that information are only given at the inspection time. Damages occurring between two consecutive inspections can not be detected which may be dangerous depending on the severity of the damage. Therefore, it is might be preferable to permanently monitor the structure by implementing sensors on it in order to have continuous information about every element of the structure. Detecting structural damages affecting structural performance, at an early stage, using sensors provides two main benefits: (i) reducing maintenance costs and facilitating a better maintenance planning since a maintenance would only be conducted when needed, (ii) increasing the lifetime of structures by preventing any catastrophic damages and taking action before it is too late. This technique would lead to a condition-based maintenance which is a cost-effective strategy avoiding the waste of lifetime provided by predetermined maintenance policy, the risk of catastrophic failure and non-scheduled maintenance while using the corrective maintenance strategy [2]. 


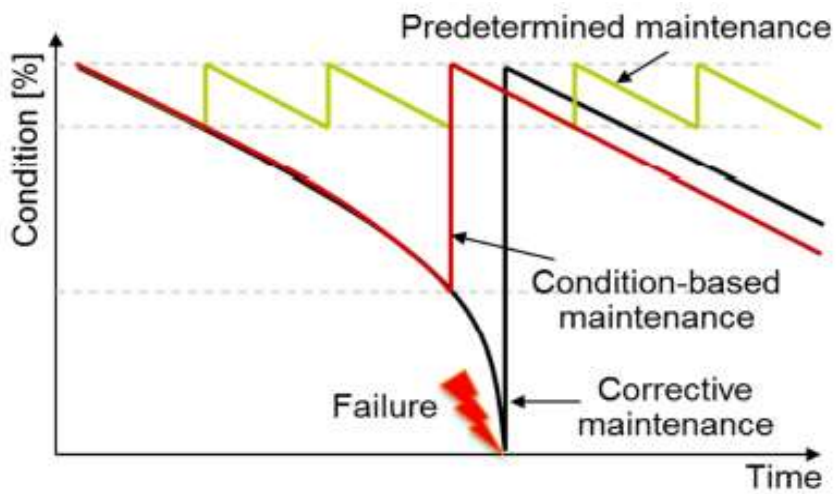

Fig. 1: Influence of the maintenance strategy on asset condition (K. Fisher and D. A. Coronado, 2015).

Most of structural health monitoring systems rely on sensors implemented on a structure to give information about only that specific structure. Since the implementation of such instruments is costly, many authors have optimized their configuration, number and location wise, in order to maximize the probability of detecting damages in a structure with a limited number of sensors [3,5]. However, in many cases, the decision maker has to optimize the configuration of sensors on a multiple similar structure (e.g. in compounds, in resorts, in schools). In such cases, one we can take advantage of the similarity by using data generated by a sensor on any of the structures to update the information for all the remaining structures and hence, optimize the configuration of sensors.

In this paper, we propose a methodology that optimizes the configuration of sensors for several similar structures by a genetic algorithm, and uses the obtained data in order to update, accordingly, the elements' properties (i.e. the rigidity, the Young modulus, etc.) for each structure through a hierarchical Approximate Bayesian Computation (ABC). This methodology considers uncertainties associated with the precision of the sensors' readings, the mechanical model and the degradation of the elements. The layout of the paper is as follows: In section 2, the proposed methodology is described, by developing the Approximate Bayesian Computation method and the genetic algorithm used. And in section 3, a numerical application on two concrete frame structures is presented to illustrate the applicability of the method.

\section{Methodology}

The methodology presented in this paper, aims at finding the optimal configuration of sensors for a group of similar structures and updating the information of the elements in any of the structures through information derived from monitoring the other structures. Thus it is divided into two main parts working simultaneously: (i) The Bayesian update of the states of each type of elements and (ii) the optimisation of the configuration of sensors through a genetic algorithm. Elements belonging to the same type are the elements having the same mechanical behaviour and subject to the same loads and environment conditions.

The steps of the proposed methodology are as follow (figure 2): (i) given a specific configuration of sensors, the data obtained from them and an a priori probability distribution of the degradation extent of a type of elements, generate $K$ random values from the degradation extent distribution, (ii) for each generated value $i$ and for each structure, postulate an $a$ priori probability distribution of the degradation extent of the structure taking into consideration the value $i$ of the degradation extent of the type of elements in question, (iii) apply the Bayesian update on the structure and calculate the Modal Assurance Criterion (MAC) defined in section 3 (Eq.3), (iv) calculate the average of the MACs calculated in (iii) defined by $\frac{\sum_{j=1}^{N} M A C}{N}$ where $N$ is the number of similar structures, (v) accept $i$ if $\frac{\sum_{j=1}^{N} M A C}{N} \leq \varepsilon$ where $\varepsilon$ is a small chosen acceptance threshold, (vi) using the accepted values, define the a posteriori distribution of the degradation of the type of elements in question, (vii) for each configuration of sensors, repeat steps (i) to (vi) for all types of elements, (viii) calculate the fitness function of the 
configuration of sensors, (ix) optimally decide if the configuration of sensors is the most effective one or try another configuration (go to step (i)) through a genetic algorithm.

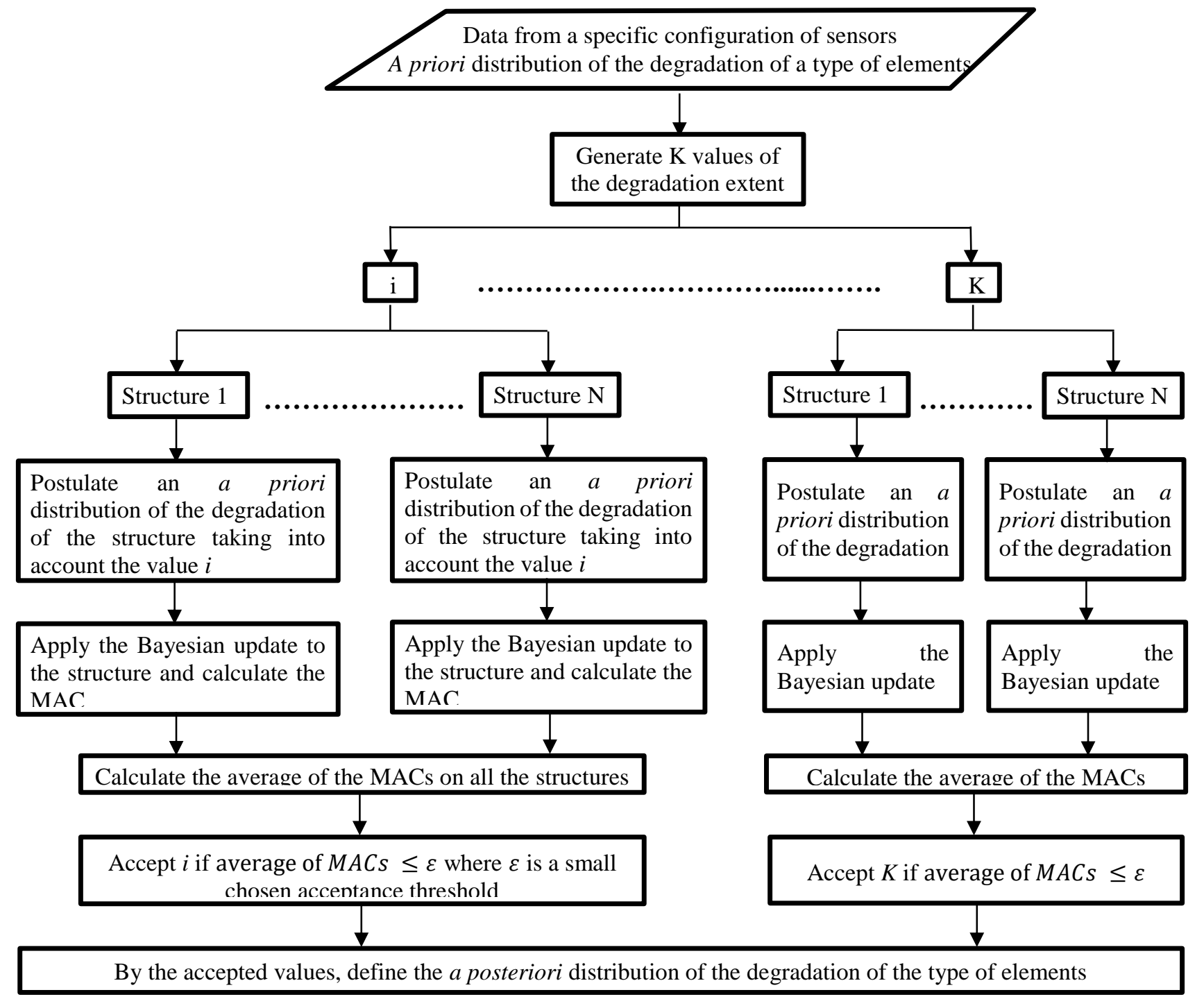

Fig. 2: Flowchart describing the Bayesian update steps for a particular type of elements and a specific configuration of sensors.

\subsection{Approximate Bayesian update (ABC)}

The aim of using the Bayesian update is to calculate an a posteriori probability distribution of the degradation extent $\beta$ of an element, after having observed the structural response $\left(\lambda_{i}^{O d}, \Phi_{i}^{O d}\right)$. The Bayesian update formula is given by:

$$
f\left(\beta \mid \lambda_{i}^{o d}, \Phi_{i}^{o d}\right)=\frac{f\left(\lambda_{i}^{O d}, \Phi_{i}^{O d} \mid \beta\right) \times f(\beta)}{f\left(\lambda_{i}^{\text {od }}, \Phi_{i}^{\text {Od }}\right)}
$$


where $\left(\beta \mid \lambda_{i}^{O d}, \Phi_{i}^{O d}\right)$ is the posterior probability distribution given the observation data $\lambda_{i}^{O d}, \Phi_{i}^{O d}, f(\beta)$ is the prior probability distribution and $f\left(\lambda_{i}^{O d}, \Phi_{i}^{O d} \mid \beta\right)$ is the likelihood function. The Bayesian update of equation (1) can be divided into a hierarchical Bayesian update as follows:

$$
\begin{gathered}
f\left(\lambda_{i}^{d}, \Phi_{i}^{d} \mid \lambda_{i}^{O d}, \Phi_{i}^{O d}\right)= \\
f\left(K^{d} \mid \lambda_{i}^{d}, \Phi_{i}^{d}\right)=\frac{f\left(\lambda_{i}^{O d}, \Phi_{i}^{O d} \mid \lambda_{i}^{d}, \Phi_{i}^{d}\right) \times f\left(\lambda_{i}^{d}, \Phi_{i}^{d}\right)}{f\left(\lambda_{i}^{O d}, \Phi_{i}^{d} \mid K^{d}\right) \times f\left(K^{d}\right)} \\
f\left(\lambda_{i}^{d}, \Phi_{i}^{d}\right) \\
f\left(\beta \mid K^{d}\right)=\frac{f\left(K^{d} \mid \beta\right) \times f(\beta)}{f\left(K^{d}\right)}
\end{gathered}
$$

where $K^{d}$ is the stiffness matrix of the damaged structure and $\left(\lambda_{i}^{d}, \Phi_{i}^{d}\right)$ is the actual response which is different from the observed structural response due to uncertainties related to the mechanical model and the accuracy of sensor measurements.

An analytical solution for this problem is generally not feasible because some of the likelihood functions are usually implicit. For example, the likelihood of equation (2b) is implicit because the relation between the structural parameters $\left(K^{d}\right)$ and the structural response $\left(\lambda_{i}^{d}, \Phi_{i}^{d}\right)$ is usually described by numerical models such as a Finite Element Model (FEM).

Thus, in our case, we considered the Approximate Bayesian Computation (ABC) algorithm [6,7] to calculate an $a$ posteriori probability distribution of the vector $\beta$.The $\mathrm{ABC}$ algorithm is essentially computed as follows:

1) Postulate an a priori distribution of the degradation extent of the structure and generate $\beta$;

2) Obtain a list of modified stiffness $K^{d}$ by multiplying each element's stiffness by its corresponding $\beta$;

3) Calculate a noisy structural response by taking into account all uncertainties through a FEM;

4) Simulate $\lambda_{i}^{S O d}, \Phi_{i}^{S O d}$

5) Compare the observed structural response $\lambda_{i}^{O d}, \Phi_{i}^{O d}$ and the simulated one $\lambda_{i}^{S O d}, \Phi_{i}^{S O d}$ using the Modal Assurance Criterion (MAC) matrix [8,9] defined by Eq.3;

The modal assurance criterion measures the correlation between the two eigenvectors $\Phi_{i}$ and $\Phi^{\prime}{ }_{i}$. The diagonal elements of the matrix MAC are calculated by the formula:

$$
M A C_{i, i}=\frac{\left[\Phi_{i}^{T} \Phi_{i}^{\prime}\right]^{2}}{\left[\Phi_{i}^{T} \Phi_{i}\right]\left[\Phi_{i}^{\prime T} \Phi_{i}^{\prime}\right]}
$$

where $i$ corresponds to the vibration mode and $\Phi_{i}$ et $\Phi^{\prime}{ }_{i}$ are limited to the degrees of freedom observed by the sensors in our case.

The real mechanical behaviour of a structure presents some eccentricities compared to the predictions of the mechanical analysis due to many factors such as: imperfections of the mathematical model, numerical approximations, etc. These uncertainties are taken into consideration by applying an initial $\mathrm{ABC}$ to the structure free of defects to update the $a$ priori distributions of the structural parameters and then, the resulting a posteriori distribution will be used as an a priori for the detection of any future damage configuration.

\subsection{Genetic algorithm}

The optimal sensor placement (OSP) is a common optimization problem that has been tackled, through the years, by many authors using genetic algorithms such as L. Yao et al. (1993) and B.K. Jung et al. (2015) [10-11]. These authors proposed several algorithms to monitor different types of single structures (e.g. large space structures, high-rise structures, 
flexible structures, etc.) however, optimizing the configuration of sensors on a set of similar structures has not been developed yet to our knowledge.

In order to detect damages in similar structures, one should judiciously distribute the sensors on the structures so that the maximum of information about all the structures is given with the minimal possible cost. For that purpose, the configuration of sensors is modelled using a genetic algorithm on a population of chromosomes representing the configurations. Each chromosome has a size $N_{s}$ equal to the number of the degrees of freedom of all the structures, with genes taking the value of 1 if the correspondent degree of freedom is observed by the sensor and 0 if not.

The loss function representing the fitness function to be minimized in the algorithm is given by the sum of squares:

$$
f=\sum_{i=0}^{n}\left(\bar{x}_{i}-x_{i}\right)^{2}
$$

Where $\bar{x}_{i}$ and $x_{i}$ are respectively the real value and the calculated value of the defect of the element $i$, and $n$ the number of elements in each structure.

The optimal configuration of sensors for similar structures would be the most accurate one, which means the one having the minimal $f$.

\section{Numerical application}

To demonstrate the applicability of the proposed methodology, we consider the case of two simply supported concrete frames, each one composed of ten rectangular elements and nine nodes which is equivalent, in total, to 21 degrees of freedom (figure 3). The elements dimensions are supposed to be $20 x 60 \mathrm{~cm}$ for the vertical ones and $20 \mathrm{x} 40 \mathrm{~cm}$ for the horizontal ones. We assume that, in both structures, the elements 1 and 10 are damaged having lost $60 \%$ of their initial modulus (33GPa), but the damages have not been detected yet. The a priori distribution of the degradation of all the elements is supposed to be uniform non informative at the beginning of the problem. This study has two main objectives: (i) find the best configuration of sensors, number and positioning wise, on both structures simultaneously taking into consideration their similarity and (ii) update the information about all the elements, in both structures, from data coming from any sensor implemented in any of the structures.

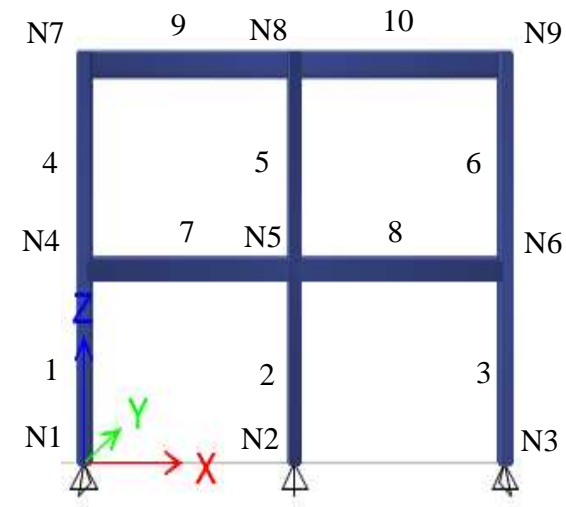

Fig. 3: The simply supported concrete frame representing the two structures in question.

The Bayesian update has been applied on 60,000 samples with an acceptance threshold value equal to 0.02. And the genetic algorithm used for the optimal configuration of sensors has been applied using 50 generations of 50 individuals each, with a population representing the sensors by chromosomes of size 42 (equal to the number of degrees-of-freedom of both structures). The optimal configuration of sensors obtained for the two structures in question consists of implementing sensors on node N4 in the first structure and node N9 in the second one. 
Some of the obtained results are represented in figures 4 and 5 showing, respectively, the posterior distributions of the degradation rate of rigidities of columns 1 and 3, and beams 8 and 10, after the defects have been detected by the sensors.

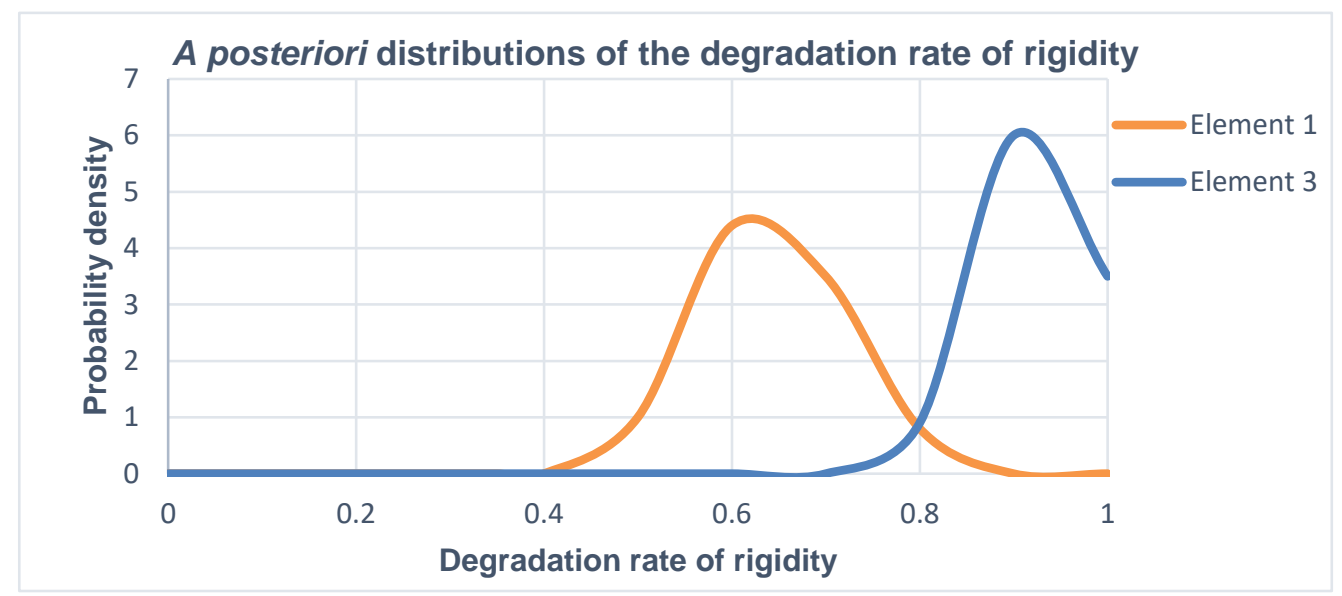

Fig. 4: Posterior distributions of the degradation rate of rigidity of element 1 and 3.

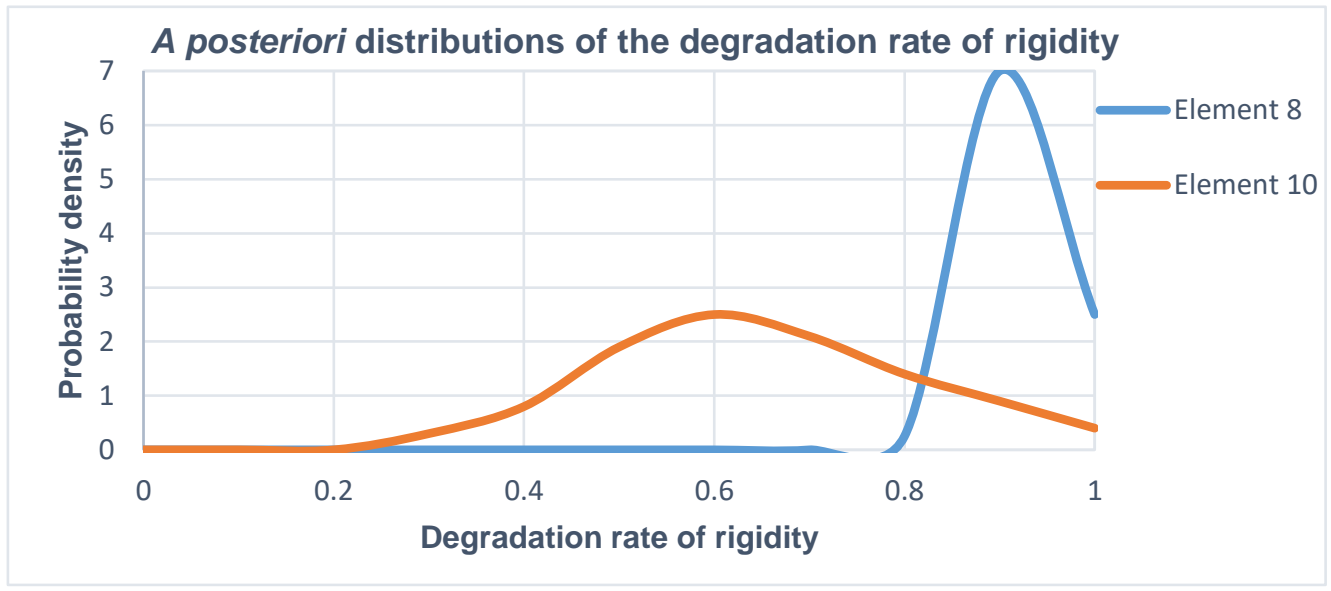

Fig. 5: Posterior distributions of the degradation rate of rigidity of element 8 and 10 .

As one can notice, the posterior distributions of column 1 and beam 10 converge to the value of 0.6 , which means that both elements are degraded. These results meet the initial hypothesis taken since elements 1 and 10 are assumed to have lost $60 \%$ of their initial modulus (unknown in reality).

On another hand, even though the posterior distributions of elements 3 and 8 show that both elements are in good conditions, they have not converged to the value 1 as one can expect however, they tend to the value 0.9 . This small difference is due to the fact that the non-informative prior distribution taken is a uniform function from 0 to 1 with a mean of 0.5 .

Furthermore, when comparing the optimal configuration of sensors obtained for two similar structures to the configuration of sensors on each structure alone, which consists on implementing on one of structure a sensor on N4 and on the second one a sensor on N9, it was found that in the first case the accuracy of the results represented by $f=0.097$ is much better than the second one where $f=0.5$. Hence, for the same number of structures and same number of sensors, results improve significantly when the similarity is taken into consideration. 
Therefore, it can be concluded that the proposed algorithm is a cost-efficient solution for the monitoring of multiple similar structures at a time.

\section{Conclusion}

In this paper, we have presented an approach to update the information about the degradation extent of elements belonging to similar structures using a global permanent SHM system and optimize the configuration of sensors to be implemented on these structures. The proposed technique consists of taking advantage of the data given by the sensors implemented on all the similar structures in order to update the state of each element in the structures using a hierarchical Approximate Bayesian Computation (ABC). This update procedure was also integrated in a genetic algorithm by choosing a near optimal sensor configuration for all structures. The benefits of this approach is that: (i) it gives the opportunity to follow the degradation extent of an element in a structure from sensors implemented in other similar structures, (ii) it gives information about a high number of elements with a low number of sensors which makes it a cost-effective method. A numerical application for a two simply supported frame structures was presented to demonstrate the applicability of the proposed methodology.

\section{Acknowledgements}

This work was supported by the National Council for Scientific Research in Lebanon (CNRS-L).

\section{References}

[1] R. Ahmad and S. Kamaruddin, "An overview of time-based and condition-based maintenance in industrial application," Comput. Ind. Eng., pp. 135-149, 2012.

[2] K. Fisher and D. A. Coronado, "Condition monitoring of wind turbines: state of the art, user experience and recommendations," Fraunhofer-IWES, Bremerhaven, 2015.

[3] C. Papadimitriou, "Optimal sensor placement methodology for parametric identification of structural systems," J. Sound Vib., vol. 278, no. 4-5, pp. 923-947, 2004.

[4] P. N. Ngatchou, W. L. Fox and M. A. El-Sharkawi, "Distributed sensor placement with sequential particle swarm optimization," in Proceedings of the IEEE International Conference Swarm Intelligence Symposiun, Pasadena, CA, 2005, pp. 385-388.

[5] L. He, J. Lian, B. Ma and H. Wang, "Optimal multiaxial sensor placement for modal identification of large structures," Struct. Control Hlth., vol. 21, no. 1, pp. 61-79, 2014.

[6] D. Wegmann, C. Leuenberger, L. Excoffier, "Efficient approximate Bayesian computation coupled with Markov chain Monte Carlo without likelihood," Genetics, vol. 182, pp. 1207-1218, 2009.

[7] R.D. Wilkinson, "Approximate Bayesian computation (ABC) gives exact results under the assumption of model error. Statistical applications in genetics and molecular biology," Stat. Appl. Genet. Mol., vol. 12, no. 2, pp. 129-141, 2013.

[8] R.J. Allemang, "The Modal Assurance Criterion (MAC): Twenty Years of Use and Abuse," J. Sound Vib., vol. 37, no. 8, pp. 14-23, 2003.

[9] M. Pastor, M. Binda, T. Harčarik, "Modal Assurance Criterion,” Procedia Eng., vol. 48, pp. 543-548, 2012.

[10] L. Yao, W.A. Sethares, D.C. Kammer, "Sensor placement for on-orbit modal identification via a genetic algorithm," AIAA J., vol. 31, no. 10, pp. 1922-1928, 1993.

[11] B.K. Jung, J.R. Cho, W.B. Jeong, "Sensor placement optimization for structural modal identification of flexible structures using genetic algorithm,” J. Mech. Sci. Technol., vol. 29, no. 7, pp. 2775-2783, 2015. 